\title{
Intense Exercise Causing Central Retinal Vein Occlusion in a Young Patient: Case Report and Review of the Literature
}

\author{
Elad Moisseiev ${ }^{\mathrm{a}}$ Oded Sagiv $^{\mathrm{b}}$ Moshe Lazar $^{\mathrm{a}}$ \\ ${ }^{a}$ Department of Ophthalmology, Tel Aviv Medical Center, Tel Aviv, and \\ ${ }^{\mathrm{b}}$ The Goldschleger Eye Institute, Sheba Medical Center, Tel Hashomer, Israel
}

\section{Key Words}

Central retinal vein occlusion $\cdot$ Macular edema $\cdot$ Bevacizumab

\begin{abstract}
We report a 19-year-old patient who developed a central retinal vein occlusion (CRVO) with significant macular edema and visual impairment following intense exercise and dehydration. The patient was treated with 3 intravitreal bevacizumab injections with complete resolution. A review of the literature on the cause and treatment for CRVO in young patients was performed, focusing on the role of intense exercise and dehydration as a rare pathogenesis mechanism of CRVO.

(c) 2014 S. Karger AG, Basel
\end{abstract}

\section{Introduction}

Central retinal vein occlusion (CRVO) is the second most common retinal vasculopathy and predominantly occurs in patients over 60 years of age with associated risk factors, such as vascular disease, hypertension and glaucoma [1]. A minority of patients with CRVO are young adults, under 40 years of age. In most of these cases, CRVO occurs without the presence of the typical risk factors, and a comprehensive workup is warranted [2]. Unusual causes for CRVO in young patients include hypercoagulability states, collagen vascular diseases, lymphoproliferative disorders, malignant hypertension, medications (most notably oral contraceptives and isotretinoin), and trauma [2-13]. A recent study has demonstrated that hyperlipidemia and hyperhomocysteinemia were the most significant risk factors for CRVO in patients under 40 [14]. 
Moisseiev et al.: Intense Exercise Causing Central Retinal Vein Occlusion in a Young Patient: Case Report and Review of the Literature

Intense exercise and associated dehydration have rarely been reported to cause CRVO in young patients [15-17]. We report a patient who suffered CRVO following intense exercise and was treated with intravitreal bevacizumab injections with complete resolution.

\section{Case Report}

A 19-year-old man was referred to our clinic due to decreased visual acuity (VA) in his left eye. The patient had no previous systemic or ocular medical history and did not use any medication. His complaint of decreased VA acutely started 1 week prior to his presentation, after several days of intense physical activity (long distance running and weight lifting performed outdoors during the summer). He remained stable during the time of exercise.

On initial examination, his best corrected VA was 20/20 in the right eye and 20/150 in the left eye. His intraocular pressure was $12 \mathrm{~mm} \mathrm{Hg}$ in both eyes, the range of ocular movements was fully bilateral, and there was no relative afferent pupillary defect. A dilated fundus examination of the right eye and both anterior segments were normal. Fundus examination of the left eye was significant for a swollen disc, numerous retinal hemorrhages, macular edema (ME) and tortuous dilated retinal vasculature (fig. 1a). Fluorescein angiography demonstrated hyperfluorescence compatible with disc hyperemia, ME, and areas of blocked fluorescense compatible with the retinal hemorrhages. Acapillary nonperfusion was not observed (fig. 1b). A significant ME was demonstrated by optical coherence tomography (fig. 1c).

Our young patient was diagnosed with CRVO. Our workup included a normal complete blood count, blood chemistry, lipid profile and coagulation tests (PT and PTT). The erythrocyte sedimentation rate was normal $(5 \mathrm{~mm} / \mathrm{h})$, and a complete collagenogram, including the rheumatic factor, antinuclear antibodies and antineutrophil cytoplasmic antibodies, was also normal. Hemoglobin electrophoresis, the complete neurological examination and the head CT were also normal, as was the further workup for an increased coagulability (including tests for hyperhomocysteinemia, antiphospholipid antibodies, factor $\mathrm{V}$ Leiden mutation and $\beta 2$-glycoprotein).

The patient was treated with 3 monthly intravitreal injections of bevacizumab (1.25 $\mathrm{mg} / 0.05 \mathrm{ml}$ ). Following these 3 injections, VA in the left eye had improved to 20/20, the hemorrhages had resorbed, and the fundus examination returned to normal (fig. 1d, e). The ME had also been completely resorbed with the restoration of a normal macular architecture (fig. 1f). The patient was observed for an additional year with no recurrence.

\section{Discussion}

The natural history of eyes with CRVO is associated with a poor visual prognosis, especially in cases with nonperfused CRVO [19, 20]. Until recently, treatment of ME in patients with CRVO had been guided by the historic Central Vein Occlusion Study that recommended mere observation in these cases [21]. However, significant advances had been made in recent years, and there are now several effective treatment options for the affected eyes. These include intravitreal injections of steroidal agents, such as triamcinolone acetate [22] and the ozurdex slow-release dexamethasone implant [23], and anti-vascular endothelial growth factor agents such as ranibizumab [24], bevacizumab [25, 26] and aflibercept [27].

In contrast to older patients, CRVO in young adults has a much more favorable natural history. At presentation, VA is typically not reduced and a spontaneous resolution over 
Moisseiev et al.: Intense Exercise Causing Central Retinal Vein Occlusion in a Young Patient: Case Report and Review of the Literature

several months is possible. However, it should be noted that some of these eyes suffer a significant and permanent visual impairment and may develop complications such as chronic ME, retinal neovascularization and vitreal hemorrhage [2]. Due to the rarity of CRVO in young patients, randomized controlled trials of the various treatment options for these patients has not yet been performed, and there are currently no established guidelines for the treatment of these patients.

Dehydration due to either fasting or intense exercise has been infrequently reported as a cause of CRVO in young patients [15-18]. Out of the 11 patients reported in these works, only 1 had been treated with bevacizumab. This was a 40 -year-old male athlete with CRVO that occurred following intense exercise and secondary dehydration. The patient was treated with 11 bevacizumab injections and he experienced significant visual improvements [17]. The patient we described had no previous medical or ocular history and underwent a comprehensive investigation that revealed no causative factor for CRVO. Since the symptoms started after a period of intense exercise, it is likely that the patient had been dehydrated and that was the cause for the occurrence of CRVO. During dehydration, the blood hematocrit increases, which results in a temporary hyperviscosity state. The central retinal vein is susceptible for thrombosis in the presence of hyperviscosity, which reduces the blood flow, especially at the point it goes through the lamina cribrosa [28]. This mechanism may explain the occurrence of CRVO secondary to intense exercise and dehydration.

We describe a second case of a young patient with CRVO, caused by intense exercise, that was treated with bevacizumab. After 3 monthly injections, a complete resolution with a restoration of 20/20 vision was achieved, and no recurrence occurred following cessation of treatment. Although it is possible that a spontaneous resolution would have occurred even without treatment in this patient, it should be noted that ME was significant and his VA was reduced in an otherwise healthy and young patient. Initiating treatment may have improved the natural history or hastened the visual recovery. Repeated intravitreal injections of antivascular endothelial growth factor agents have been established as a safe and effective treatment method for ME in CRVO [24-26] and may very well be beneficial in young patients with CRVO.

This case report should raise awareness to the possibility of intense exercise as a possible cause for CRVO in young patients. In the absence of an established treatment regimen for these cases, we suggest that treatment can be initiated despite the possibility of a spontaneous resolution. Intravitreal bevacizumab injections seem to be a safe and effective treatment option for such cases.

\section{Disclosure Statement}

The authors declare that there are no conflicts of interest regarding the publication of this article.

\section{References}

1 The Eye Disease Case-Control Study Group: Risk factors for central retinal vein occlusion. Arch Ophthalmol 1996;114:545-554.

-2 Fong ACO, Schatz H: Central retinal vein occlusion in young adults. Surv Ophthalmol 1993;37:393-417.

-3 Bharathi Devi SR, Suganeswari G, Sharma T, Thennarasu M, Angayarkanni N: Homocysteine induces oxidative stress in young adult central retinal vein occlusion. Br J Ophthalmol 2012;96:1122-1126.

4 Mouinga Abayi DA, Giraud JM, Fenolland JR, et al: A rare trauma-associated cause of central retinal vein occlusion in a young subject (article in French). J Fr Ophtalmol 2012;35:426-431. 


\begin{tabular}{l|l}
\hline \multicolumn{2}{l}{ Case Rep Ophthalmol 2014;5:116-120 } \\
\hline DOI: 10.1159/000360904 & $\begin{array}{l}\text { ○ 2014 S. Karger AG, Basel } \\
\text { www.karger.com/cop }\end{array}$ \\
\hline
\end{tabular}

Moisseiev et al.: Intense Exercise Causing Central Retinal Vein Occlusion in a Young Patient: Case Report and Review of the Literature

5 Parvin P, Pournaras JA, Wolfensberger TJ: Importance of complete haematological workup for relatives of young patients presenting central retinal vein occlusion. Klin Monbl Augenheilkd 2011;228:383-384.

-6 Balogh Z, Berta A, Pfliegler G, Nagy V: Bilateral central retinal vein occlusion caused by malignant hypertension in a young patient. Clin Exp Hypertens 2011;33:53-55.

7 Rehak M, Krcova V, Slavik L, et al: The role of thrombophilia in patients with retinal vein occlusion and no systemic risk factors. Can J Ophthalmol 2010;45:171-175.

-8 Thapa R, Paudyal G: Central retinal vein occlusion in young women: rare cases with oral contraceptive pills as a risk factor. Nepal Med Coll J 2009;11:209-211.

-9 Tseng MY, Chen YC, Lin YY, Chu SJ, Tsai SH: Simultaneous bilateral central retinal vein occlusion as the initial presentation of acute myeloid leukemia. Am J Med Sci 2010;339:387-389.

10 Kuhli-Hattenbach C, Scharrer I, Lüchtenberg M, Hattenbach LO: Coagulation disorders and the risk of retinal vein occlusion. Thromb Haemost 2010;103:299-305.

11 Jaksic V, Markovic V, Milenkovic S, Stefanovic I, Jakovic N, Knezevic M: MTHFR C677T homozygous mutation in a patient with pigmentary glaucoma and central retinal vein occlusion. Ophthalmic Res 2010;43:193196.

12 Labiris G, Katsanos A, Karapetsa M, Mpanaka I, Chatzoulis D: Association between isotretinoin use and central retinal vein occlusion in an adolescent with minor predisposition for thrombotic incidents: a case report. J Med Case Rep 2009;3:58.

13 Parodi MB, Di Crecchio L: Hyperhomocysteinemia in central retinal vein occlusion in young adults. Semin Ophthalmol 2003;18:154-159.

14 Kuo JZ, Lai CC, Ong FS, et al: Central retinal vein occlusion in a young Chinese population: risk factors and associated morbidity and mortality. Retina 2010;30:479-484.

-15 Francis PJ, Stanford MR, Graham EM: Dehydration is a risk factor for central retinal vein occlusion in young patients. Acta Ophthalmol Scand 2003;81:415-416.

-16 Alghadyan AA: Retinal vein occlusion in Saudi Arabia: possible role of dehydration. Ann Ophthalmol 1993;25:394-398.

17 Jacobs DJ, Ahmad F, Pathengay A, Flynn HW Jr: Central retinal vein occlusion after intense exercise: response to intravitreal bevacizumab. Ophthalmic Surg Lasers Imaging 2011;42:e59-e62.

18 Rouhani B, Mandava N, Olson JL: Central retinal vein occlusion after intense exercise in healthy patients. Retin Cases Brief Rep 2010;4:105-108.

19 Hayreh SS, Podhajsky PA, Zimmerman MB: Natural history of visual outcome in central retinal vein occlusion. Ophthalmology 2011;118:119-133.

20 McIntosh RL, Rogers SL, Lim L, et al: Natural history of central retinal vein occlusion: an evidence-based systematic review. Ophthalmology 2010;117:1113-1123.

-21 The Central Vein Occlusion Study Group: Evaluation of grid pattern photocoagulation for macular edema in central vein occlusion. The Central Vein Occlusion Study Group M report. Ophthalmology 1995;102:14251433.

22 Ip MS, Scott IU, VanVeldhuisen PC, SCORE Study Research Group, et al: A randomized trial comparing the efficacy and safety of intravitreal triamcinolone with observation to treat vision loss associated with macular edema secondary to central retinal vein occlusion: the Standard Care vs Corticosteroid for Retinal Vein Occlusion (SCORE) study report 5. Arch Ophthalmol 2009;127:1101-1114.

-23 Haller JA, Bandello F, Belfort R Jr, Ozurdex GENEVA Study Group, et al: Dexamethasone intravitreal implant in patients with macular edema related to branch or central retinal vein occlusion twelve-month study results. Ophthalmology 2011;118:2453-2460.

24 Brown DM, Campochiaro PA, Singh RP, CRUISE Investigators, et al: Ranibizumab for macular edema following central retinal vein occlusion: six-month primary end point results of a phase III study. Ophthalmology 2010;117:1124-1133.

25 Axer Siegel R, Dotan A, Mimouni K, et al: Intravitreous bevacizumab treatment for macular edema due to central retinal vein occlusion. Curr Eye Res 2012;37:818-822.

-26 Lu Y, Su L, Xu X: Bevacizumab for macular edema secondary to retinal vein occlusion: a systematic review and meta-analysis. J Ocul Pharmacol Ther 2013;29:702-708.

27 Brown DM, Heier JS, Clark WL, et al: Intravitreal aflibercept injection for macular edema secondary to central retinal vein occlusion: 1-year results from the phase 3 COPERNICUS study. Am J Ophthalmol 2013;155:429-437.

28 Williamson TH: A 'throttle' mechanism in the central retinal vein in the region of the lamina cribrosa. $\mathrm{Br} \mathrm{J}$ Ophthalmol 2007;91:1190-1193. 
Moisseiev et al.: Intense Exercise Causing Central Retinal Vein Occlusion in a Young Patient: Case Report and Review of the Literature
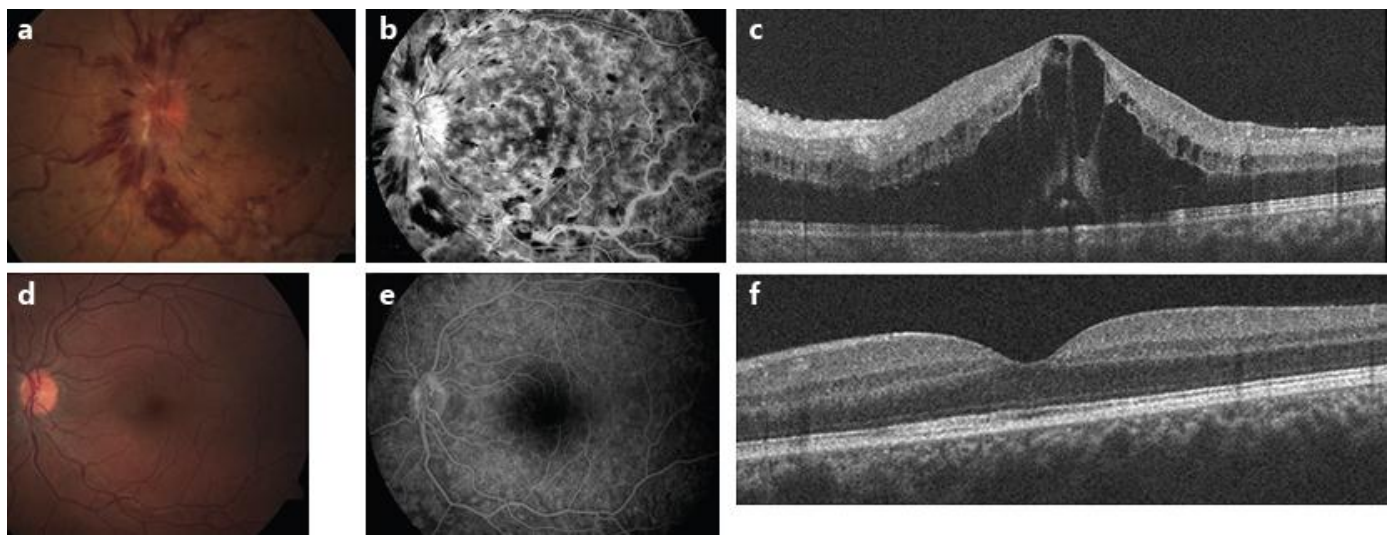

Fig. 1. At presentation, a diagnosis of CRVO was made based on the ocular findings, which included a swollen disc, multiple retinal hemorrhages and tortuous, dilated retinal vessels (a). Fluorescein angiography demonstrated disc hyperemia, ME and blocked hypofluorescence compatible with the hemorrhages (b). Optical coherence tomography demonstrated significant ME with subretinal fluid (c). Following treatment with 3 bevacizumab injections, the ocular findings had disappeared, and the fundus examination and fluorescein angiography returned to normal $(\mathbf{d}, \mathbf{e})$. The macular edema had resorbed completely and normal macular architecture was restored (f). 